University of Nebraska - Lincoln

DigitalCommons@University of Nebraska - Lincoln

\title{
Comment on "Destructive Effect of Disorder and Bias Voltage on Interface Resonance Transmission in Symmetric Tunnel Junctions"
}

Julian P. Velev

University of Nebraska-Lincoln, julian.velev@unl.edu

Kirill D. Belashchenko

University of Nebraska-Lincoln, belashchenko@unl.edu

Evgeny Y. Tsymbal

University of Nebraska-Lincoln, tsymbal@unl.edu

Follow this and additional works at: https://digitalcommons.unl.edu/cmrafacpub

Part of the Nanoscience and Nanotechnology Commons

Velev, Julian P.; Belashchenko, Kirill D.; and Tsymbal, Evgeny Y., "Comment on "Destructive Effect of Disorder and Bias Voltage on Interface Resonance Transmission in Symmetric Tunnel Junctions"'" (2006). Faculty Publications from Nebraska Center for Materials and Nanoscience. 34.

https://digitalcommons.unl.edu/cmrafacpub/34

This Article is brought to you for free and open access by the Materials and Nanoscience, Nebraska Center for (NCMN) at DigitalCommons@University of Nebraska - Lincoln. It has been accepted for inclusion in Faculty Publications from Nebraska Center for Materials and Nanoscience by an authorized administrator of DigitalCommons@University of Nebraska - Lincoln. 
Comment on "Destructive Effect of Disorder and Bias Voltage on Interface Resonance Transmission in Symmetric Tunnel Junctions"

In a recent Letter Tusche et al. [1] showed that a complete and coherent $\mathrm{FeO}$ layer forms on both interfaces of a $\mathrm{Fe}|\mathrm{MgO}| \mathrm{Fe}(001)$ magnetic tunnel junction (MTJ) when using oxygen-assisted growth. Their ab initio model for these MTJs predicted tunneling magnetoresistance (TMR) of several thousand percent due to the contribution from interface resonances (IRs) perfectly matched at the two interfaces. In this Comment, we show that in practice the predicted giant TMR is unfeasible because the IRs are mismatched by structural disorder and/or by applied bias voltage resulting in a moderate TMR.

IRs are interfacial electronic states whose weak coupling to bulk states controls their intrinsic damping, $\gamma_{0}$. IRs produce large tunneling current in MTJs if they match identical resonances at the opposite interface [2]. Structural disorder leads to additional damping $\gamma$ and, if $\gamma \gg \gamma_{0}$, smears out the IRs over an area of the interface Brillouin zone (IBZ) proportional to $\gamma / \gamma_{0}$. This reduces the $\mathbf{k}_{\|}$-resolved transmission at resonance by a factor of $\left(\gamma / \gamma_{0}\right)^{2}$ and the total conductance by a factor of $\gamma / \gamma_{0}$ [3]. Since $\gamma_{0}$ may be very small compared to $\gamma$, the effect of disorder on the IR transmission may be significant.

We demonstrate the destructive effect of disorder on TMR in a symmetric $\mathrm{Fe}|\mathrm{FeO}| \mathrm{MgO}|\mathrm{FeO}| \mathrm{Fe}(001)$ MTJ with 5 monolayers of $\mathrm{MgO}$. We perform first-principles calculations using the approach of Ref. [4] and a $800 \times$ 800 mesh of $\mathbf{k}_{\|}$points. The structural parameters are taken from Ref. [5]. Disorder is introduced by adding a small imaginary part to the energy, $\gamma$.

Figure 1 shows the resulting conductance and TMR as a function of $\gamma$. For weak disorder $\left(\gamma \ll \gamma_{0} \sim 10^{-6}\right.$ Ry) we find, in agreement with Ref. [1], a giant TMR of almost $7000 \%$. This stems from the conductance for the parallel (P) configuration, $G_{\mathrm{P}}=G_{\uparrow}+G_{\downarrow}$, being greater by two orders in magnitude than the conductance for the antiparallel (AP) configuration, $G_{\mathrm{AP}}=2 G_{\uparrow \downarrow}$. With increasing $\gamma$ the $G_{\mathrm{P}}$ decreases dramatically, and for $\gamma \gg \gamma_{0}$ TMR drops by a factor of 50 to about $140 \%$.

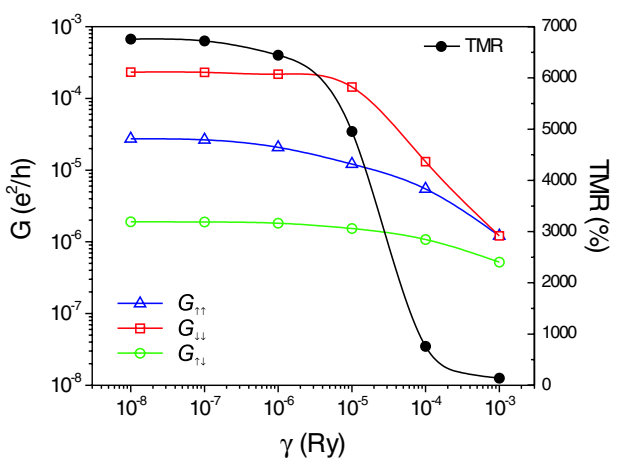

FIG. 1 (color online). Spin-resolved conductance (open symbols) and TMR (solid symbols) vs disorder parameter $\gamma$.
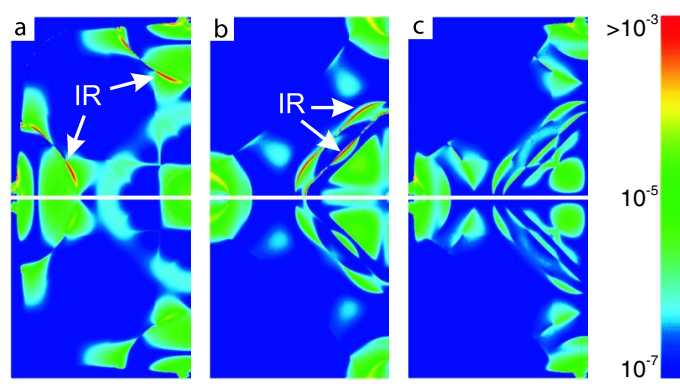

FIG. 2 (color online). $\quad \mathbf{k}_{\|}$-resolved transmission in a quarter of the IBZ for $\gamma=10^{-8}$ (upper panels) and $\gamma=10^{-3}$ Ry (lower panels). (a) Majority- and (b) minority-spin channels in the $\mathrm{P}$ configuration; (c) either spin channel in the AP configuration.

The giant TMR and its drop with disorder are the consequence of IRs formed at the two interfaces. Figure 2 shows the $\mathbf{k}_{\|}$and spin-resolved transmission for $\gamma=$ $10^{-8}$ and $\gamma=10^{-3}$ Ry [6]. The IRs are seen in the upper panels of Figs. 2(a) and 2(b) as extremely narrow lines. For $\gamma \ll \gamma_{0}$ they transmit with probability close to unity producing most of the tunneling current in the parallel configuration. With increasing $\gamma$ up to $10^{-3}$ Ry the maximum transmission value falls from 1 to about $10^{-4}$ and the overall transmission in the $\mathrm{P}$ configuration is reduced by two orders in magnitude. The transmission in the AP configuration is much less affected by disorder [Fig. 2(c)] due to a mismatch in the IRs resulting from dissimilar electronic structure at the two interfaces.

A similar effect is produced by a small applied bias voltage. Using a method of Ref. [4] we find that a bias voltage of $10 \mathrm{mV}$ is sufficient to mismatch completely the IRs in the parallel configuration resulting in a drop of TMR by two orders in magnitude (from $7000 \%$ to $72 \%$ ).

Thus, both structural disorder and applied bias voltage destroy the resonant interface transmission making giant TMR in $\mathrm{Fe}|\mathrm{FeO}| \mathrm{MgO}|\mathrm{FeO}| \mathrm{Fe}(001)$ MTJs unfeasible.

This work was supported by NSF (Grants No. DMR0213808 and No. DMR-0203359) and the NRI.

\section{J. P. Velev, K. D. Belashchenko, and E. Y. Tsymbal Department of Physics and Astronomy University of Nebraska-Lincoln Lincoln, Nebraska 68588, USA}

Received 29 November 2005; published 21 March 2006 DOI: 10.1103/PhysRevLett.96.119601

PACS numbers: 68.35.Ct, 61.10.-i, 75.70.Ak

[1] C. Tusche et al., Phys. Rev. Lett. 95, 176101 (2005).

[2] O. Wunnicke et al., Phys. Rev. B 65, 064425 (2002).

[3] E. Y. Tsymbal et al., cond-mat/0511663.

[4] K. D. Belashchenko et al., Phys. Rev. B 72, 140404(R) (2005).

[5] X.-G. Zhang et al., Phys. Rev. B 68, 092402 (2003).

[6] $\gamma=10^{-3}$ Ry provides a correct order of magnitude for low-temperature conductivity of a metallic thin film. 